\title{
UNA REFLEXIÓN CURRICULAR SOBRE LA ENSENANZA DE LA ESTRUCTURA DE LA SUSTANCIA EN LA FORMACIÓN DE PROFESORES DE QUÍMICA
}

\author{
FERRO, V.R., GONZÁLEZ-JONTE, R. H. y CRUZ, Z. \\ Instituto Superior Pedagógico. Pinar del Río 20 200. Cuba.
}

\section{SUMMARY}

In this articie we reconsider the manner of teaching the concept of structure of substance in order to improve the teaching performance of chemistry teachers. In order to achieve this goal we modify the habitual, static presentation of substance to relate it to thermodynamic and kinetic aspects. In this way we facilitate a better understanding of the chemical change.

\section{INTRODUCCIÓN}

El logro de un cambio conceptual y metodológico en la enseñanza de la química y en la formación de profesores de esta especialidad que satisfaga las exigencias del modelo del cambio conceptual (Hewson, 1981 y Segura, 1991) depende mucho de la solución que se encuentre al problema del tratamiento de los contenidos sobre la estructura de la sustancia, por la significación predominante que ellos tienen dentro del sistema de conocimientos químicos.

La dificultad de esta temática está determinada, en primer lugar, por su complejidad conceptual intrínseca -esto es la complejidad de su objeto de estudio- (Hoffmann, 1993) y por el aceierado desarrollo que experimenta esta rama del conocimiento en los últimos años (Riera, 1989).

Los didactas de la química se esfuerzan por resolver las dificultades que plantea el estudio del aspecto estructural de la reacción química y de su método de investigación; pero todavía no se puede asegurar que el propósito haya sido cumplido (Ogilvie, 1990 y Pauling 1992). La diversidad cualitativa de sistemas y planes de estudio, concepciones respecto a la enseñanza de dichos contenidos, etc. obliga a variadas y nuevas proposiciones curriculares que además deben lidiar con la ya referida complejidad creciente de la disciplina científica, tanto en su aspecto conceptual como metodológico.

En el presente trabajo se desarrollan algunas ideas en relación con la enseñanza de los contenidos sobre estruc- tura de la sustancia, en un proyecto para la formación de profesores; éstas culminan con una propuesta curricular adecuada a las exigencias que plantea la preparación de docentes capacitados para enseñar química en la época de las grandes conquistas científicas en el campo de la estructura atómica y molecular.

Las ideas que se exponen y la propuesta curricular que las materializa se contextualizan dentro de los esfuerzos por lograr la formación de un cuadro químico del mundo (CQM) en la cabeza de los estudiantes (Ferro Fernández y González-Jonte, 1992), con lo cual se pretende -traducido como una intención didáctica- la sistematización renovadora de los contenidos químicos durante la enseñanza y el aprendizaje de la química.

\section{FUNDAMENTOS DE LA PROPUESTA}

La proposición que se expone en este trabajo está basada en los siguientes criterios.

\section{La diferenciación entre las contribuciones conceptual y metodologica de los contenidos de enseñanza}

En la enseñanza de las ciencias es particularmente importante diferenciar entre el tratamiento de los conocimientos científicos de la disciplina de que se trate y la 
enseñanza de su armazón metodológico-de sus métodos de investigación-, independientemente de la profunda y compleja relación que existe entre tales cuestiones. Esto es, entre el conocimiento de los diferentes aspectos de su objeto de estudio y de los métodos que se utilizan para investigarlo.

En la impartición de la química en las aulas universitarias de casi todo el mundo -y especialmente de la formación de especialistas químicos-es práctica de no menos de 40 años la búsqueda de un fundamento estruc. tural, termodinámico y cinético en el estudio de la reacción química (Sanderson, 1960; Roberts, 1969; Karapetians y Drakin 1974; Cartmell y Fowles 1975 y Krasovitskaya 1980). La cuestión que se plantea es impartir los contenidos con un fundamento estructural, termodinámico y cinético; con lo cual se pretende significar el sentido metodológico de dichos elementos.

La distinción entre los aspectos conceptuales y metodológico de los contenidos es de vital importancia para establecer la proyección y el sentido de su tratamiento en el aula. En el modelo didáctico de la formación de un cuadro químico del mundo en los estudiantes, se insiste en dar una proyección metodológica al sistema de cono. cimientos, de manera que los estudiantes puedan pasar de las formulaciones generales de los núcleos conceptuales (Ferro Fernández y González-Jonte, 1992) a los conocimientos particulares. Una de las vias para lograr esto es el acercamiento de la lógica de las disciplinas de enseñanza a la lógica de la propia ciencia, incorporando los métodos de investigación de las ciencias químicas -el estructural, el termodinámico y el cinético- al sistema de núcleos conceptuales.

No pocos ensayos se han cumplido en este sentido, pero sólo en muy contadas ocasiones con éxito. Muchas veces el tratamiento de Ios contenidos sobre el aspecto estructural de la reacción quimica -y esto ocurre también para el termodinámico y el cinético-con pretensiones metodológicas no logra satisfacer el propósito, reduciéndose a su significado conceptual-como simple conocimiento. Una de las causas más frecuentes de este hecho es el carácter limitado con que se desarrolla la ejemplifica. ción đe los diferentes problemas conceptuales sin establecer a través de ella la contradicción y la distinción entre lo general (y su significación metodológica) y lo particular (con su significado conceptual).

\section{La diferenciación del contenido de las diferentes disciplinas del plan de estudio}

La distinción anterior fundamenta la definición de contenidos de las diferentes disciplinas curriculares en la formación de especialistas curriculares en la formación de especialistas químicos propuesta en el modelo didáctico de la formación de un cuadro químico del mundo en los estudiantes (Ferro Fernández y González-Jonte, 1992). Según este esquema, la química general y la química física son las disciplinas directamente comprometidas con el estudio de los problemas estructurales, tanto en su condición conceptual (aspecto de la reacción química) como metodológica (método de investigación de la química).

La química general debe servir de marco para la familiarización de los estudiantes con el objeto de estudio de la química-la sustancia: su estructura y estados de existencia; y la reacción química: sus aspectos y formas de realización- (Ferro Fernández y González-Jonte, 1992). En ella lo estructural, lo termodinámico y lo cinético observan la condición de aspectos de la reacción quimica (y, por tanto, en el proceso docente tienen sentido conceptual) y no de métodos.

La química física, por su parte, debe pertrechar a los estudiantes con los métodos de investigación en la química. Aquí los contenidos de enseñanza deben tenet una proyección metodológica; éste es el momento en que los métodos de investigación de la ciencia química se insertan en el esquema curricular de carrera. La división de la química física en tres asignaturas responde a la necesidad de revelar las particularidades de los métodos estructural (Q.F. I), termodinámico (Q.F II) y cinético (Q.R. III).

\section{La distinción entre los métodos clásico y cuántico en el estudio de la estructura de la sustancia}

En el presente artículo, la diferencia entre los métodos cuántico y clásico se abordará sólo en su expresión didáctico-metodológica. Estudios realizados con una muestra representativa del alumnado de la carrera profesoral de química, del Instituto Superior Pedagógico de Pinar del Río (Ferro Fernández, 1994) permitieron establecer que:

a) Los estudiantes no son capaces de diferenciar las esencias de los enfoques clásico y cuántico en el estudio de la estructura de la sustancia, sino que predomina una comprensión de carácter sincrético, no diferenciada, donde se mezclan explicaciones clásicas y cuánticas sin la observancia de la abrumadora distancia que los separa. La expresión más alta de este hecho, por sus consecuencias negativas en la formación de un correcto cuadro químico del mundo en los estudiantes, es la no distinción de las causas del enlace -covalente- según las concepciones clásicas y cuánticas.

b) Las dificultades en el aprendizaje de los estudiantes (señaladas en el punto anterior) tienen su antecedente en el proceso de enseñanza. En la literatura docente y las conferencias de los profesores se observa una pobre diferenciación conceptual y metodológica de los métodos clásico y cuántico de estudio de la estructura de la sustancia. En relación con los libros de texto sucede lo que caracteriza Izquierdo Aymerich (1992) ea los siguientes términos:

«[...] se concede más importancia a la exactitud del contenido que a lo que el libro puede llegar a comunicar al lector [...] Muy a menudo los alumnos creen entender el libro pero en realidad no saben a qué se refiere, porque 
no pueden relacionar su contenido con referentes externos a él[...]”

c) Al sincretismo clásico-cuántico (ya referido), que se manifiesta en los estudiantes durante el estudio estructural de la sustancia y la reacción química contribuye la disciplina de química general y su manera de impartición de los contenidos. Es decir, aquí se presentan nociones conceptuales -como contenido-sobre estructura atómica y molecular desde el punto de vista cuántico sin que el estudiante lo haya visto -como método de estudio del micromundo- en su génesis y evolución formal estructural; de modo que, para el estudiante, la idea de los orbitales, Ios números cuánticos, el espín, etc. queda despojada de su "plataforma» metodológica sin la cual se pierde la comprensión meridiana del asunto. Por otro lado, se comete el error (según este análisis) de no tratar otros temas referidos a la estructura de la sustancia (como la agregación molecular y el estado sólido, por ejemplo; generalmente se resuelven de forma clásica en los cursos para la formación de profesores), por incluir el tratamiento cuántico del átomo y el enlace químico; quedando todo a medias: de una parte, el estudio de la estructura en toda su diversidad fenomenológica y, de otra, el enfoque cuántico de las cuestiones estructurales.

Si en la formación de químicos (no se trata de química general para otras especialidades) y según la idea desarrollada en el punto anterior, corresponde a la química general estudiar lo estructural como aspecto de la reacción; entonces puede -perfectamente-resolverse la tarea de forma clásica y de manera completa (que es aquí lo importante): desde el átomo hasta la interacción entre moléculas y renunciando al empeño de una aproximación cuántica, limitada como contenido a unos pocos problemas y completamente «descabezada» como método. El estudio del átomo puede conducirse hasta el modelo transicional de Bohr-Sommerefeld con el cual aparecen los dos números cuánticos suficientes para intentar el estudio de la ley y tabla periódica en su generalidad y consecuencias.

Esta posición es coincidente por el criterio expuesto por Pauling (1992), al evaluar las implicaciones de la adopción del método cuántico para el estudio de enlace en la enseñanza de la química:

«Beginning courses in Chemistry ${ }^{1}$ should emphasize the simpler aspects of molecular structure in relation to the properties of substances. These aspects include the electronic structures of atoms, with emphasis on the noble-gas structure, the shared electron-pair bond [...] the electronegativity scale, partial ionic character of bonds [...] some things, especially molecular orbitals should be left out, but [...] the classical concept of the chemical bond [...] must be included in the course[...]"

\section{PRESENTACIÓN DE LA PROPUESTA}

La propuesta del presente trabajo se resume, en su expresión conceptual y su intención metodológica, en
Ios esquemas de contenido para los tratamientos clásico y cuántico de las cuestiones sobre la estructura de la sustancia que se presentan en los anexos I y II respectivamente. En algunos casos, la identificación de los contenidos tiene carácter explicativo, pero ello responde a la decisión de los autores de resolver a través de tos esquemas de contenido las cuestiones metodológicas particulares respecto a su tratamiento; esto es, las ideas de carácter metodológico relacionadas con Ia forma de explicar los contenidos están implícitas tanto en su enunciación como en el ordenamiento en que se presentan.

A continuación se precisan aigunas ideas de significación didáctico-metodológica general respecto a la realización de la propuesta curricular desarrollada:

1. El problema de la estructura de la sustancia se resuelve, desde sus inicios, reconociendo la interacción entre las partículas como una forma particular del movimiento del micromundo; idea que debe conducir el estudio del enlace químico, en contraposición a la percepción generalizada, entre los estudiantes, del reposo y de la inercia respecto al estado estructural estable en las especies moleculares.

De esta manera se puede presentar sistemáticamente -en relación con la mecánica, Ia termodinámica, etc.- el concepto de energía de enlace y energía química, que usualmente se hace tan difícil a la comprensión de los estudiantes.

2. En el modelo didáctico de la formación de un CQM en los estudiantes (Ferro Fernández y González-Jonte, 1992) que sustenta esta propuesta, se ensaya la presentación de los nuevos contenidos en torno al concepto de reacción química. De esta manera el estudio de la estructura de la sustancia debe -porque objetivamente existe tal determinación- revelar el aspecto estructural de la reacción química.

Un detalle a significar en este esfuerzo es que el estudio de las cuestiones geométrico-estructurales de la sustancia alcanza sentido porque cambia por la existencia de los cambios químicos y químico-físicos que la modifican. Sin embargo, se hace formalmente difícil poner de manifiesto este hecho con auxilio del aparato conceptual y metodológico en que descansa el ejercicio docente que se desarrolla en las aulas de pregrado. Estructura de la sustancia y reacción química parecen ser dos problemas diferentes relacionados: el primero, con el cambio, el movimiento y la dinámica, en la sustancia.

La solución a este aparente contradicción puede estar en presentar la interacción a niveles atómico molecular e intermolecular como una forma específica de movimiento, en estrecha relación con la estructura de la sustancia. Desde esta posición puede comprenderse que el estructuralismo en el mundo material (entendido como la existencia objetiva de un arreglo estructural en los sistemas materiales) responde al hecho de que la existencia de fuerzas opuestas en cualquiera de sus niveles de complejidad impone un ordenamiento particular de los elementos sustanciales para cada estado de correlación 
entre esas fuerzas. La estructura es -entonces- el ordenamiento de los elementos sustanciales para cada estado de movimiento del sistema material como un todo. La «cualidad química» ligada a cada uno de esos estados es la naturaleza química de la sustancia en cuestión, y los cambios que ella experimenta -por su parte-, la reacción química. La reacción es un proceso tiempo-dependiente, pero la evolución es tal que, para cada instante, existe un ordenamiento estructural concreto (estable o no, pero existente) como consecuencia de la inagotabilidad de la materia. Al hombre le es posible «fijar" y reproducir sólo algunos de esos estados (los más estables); por lo tanto, cada uno de los estados estructurales que se nos revelan como sustancias químicas específicas constituyen un instante -y un estado-del evolucionar cualitativo de la materia.

Así se puede definir que la estructura es el aspecto formal del movimiento químico de la materia cuya expresión espacial (en el espacio) es la geometría molecular.

En el formalismo de la química cuántica es amplia y predominantemente utilizado el modelo t-independiente de Schrödinger, desarrollado sobre especies estables. La descripción de la reacción química debe hacerse, dentro de estos marcos, como una sucesión de estados t..jndependientes.

Para superar toda esta complejidad se debe relacionar el estudio de la reacción con su aspecto estructural y acompañar siempre la descripción del estado estructural de la evaluación de la transformación que potencialmente to puede modificar.

3. El reconocimiento de la determinación de la estructura sobre las propiedades de la sustancia tiene carácter rector en el estudio de la química (Kuznetzova, 1985; Ferro Fernández y González-Jonte, 1992). Ello, unido a la aceptación de tres niveles de complejidad en la organización estructural de la sustancia (atómico, molecular e intermolecular) debe constituir un principio jerárquicamente predominante en la selección y explicación de los contenido (Esquema 1).

Observar este esquema en la estructuración molecular y la presentación de los contenidos evita dificultades en el aprendizaje de los estudiantes, como las siguientes, las cuales han sido constatadas por los autores en su ejercicio docente:
NIVEL ATÓMICO<smiles>[3H]I</smiles>

NIVEL MOLECULAR

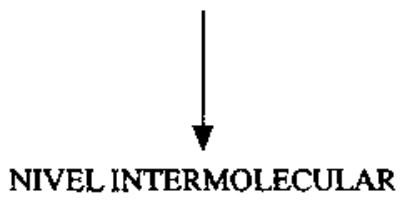

NIVEL INTERMOLECULAR

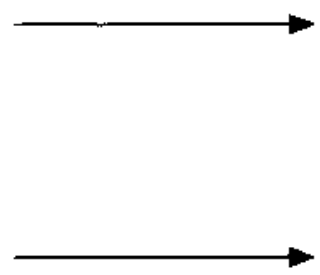

PROPIEDADES QUÍMICAS DE LOS ELEMENTOS
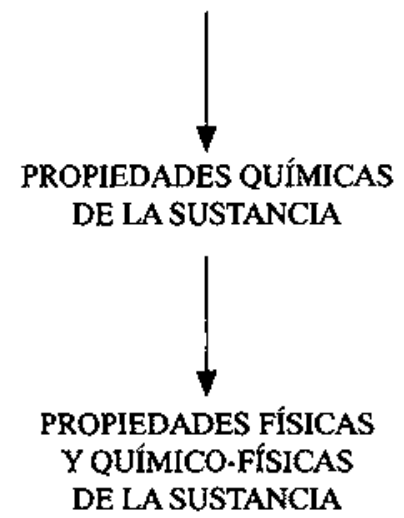
- ubicar sustancias simples diatómicas $\left(\mathrm{Cl}_{2}\right)$ en la tabla periódica;

- relacionar la diferencia entre las temperaturas de fusión i ebullición de dos sustancias $\left(\mathrm{SiF}_{4}\right.$ y $\left.\mathrm{NaF}\right)$ con la naturaleza del enlace que une a sus átomos en la estructura «molecular».

\section{CONSIDERACIONES FINALES}

En este artículo se han presentado los fundamentos de una propuesta curricular para la enseñanza de la estructura de la sustancia en un programa de formación de profesores de química. Se precisaron los elementos más

\section{REFERENCIAS BIBLIOGRÁFICAS}

CARTMELL, E. y FOWLES, G.W.A. (1975). Valencia y estructura molecular. Barcelona: Reverté.

FERRO FERNÁNDEZ, V.R. y GONZÁLEZ-JONTE, R.H. (1992). La formación de un ctradro químico del mundo en los estudiantes como modelo para la sistematización de los contenidos quimicos. Studia Chemica. Sección Didáctica, 17, pp. 127-136.

FERRO FERNÁNDEZ, V. R. (1994). Propuesta didactica para el tratamiento de los problemas sobre estructura de la sustancia en el Plan C de Formación de Químicos en los institutos superiores pedagogicos. Ponencia Presentada en Ia Reunión Metodológica del Departamento de Química del Instituto Superior Pedagógico de Pinar del Río. Pinar del Río.

HEWSON, P.W. (1981). A Conceptual Change Approach to Learning Science. European Journal of Science Education, 3(4), pp. 383-396.

HOFFMAN, R. (1993). Seeking Beauty in Atoms. New York Times, July 6.

IZQUIERDO AYMERICH, M. (1992), ¿A qué se refieren los textos de química? II Coloquio Internacional sobre la Enseñanza Superior de la Química en Lenguas Internacionales de Origen Latino. Málaga.

KARAPETIANS, M. J. y DRAKIN, S. I. (1974). Estructura de la sustancia. Moscú: MIR. generales (de carácter diđáctico-metodológico y conceptual) de su realización, dejando para un trabajo posterior el análisis de los resultados de su aplicación con carácter experimental a un grupo de estudiantes.

\section{NOTA}

'Los autores consideran la química general como uno de los «cursos iniciales» en química que menciona L. Pauling.

KARAPETIANS, M. J. y DRAKIN, S. I. (1974). La estructura electrónica de los átomos y el enlace químico. Moscú: Provishenie.

KUZNETZOVA, N.E. (1985). Formación de sistema de conocimientos en la enseñanza moderna de la química. : Leningrado: Editora del Instituto Pedagógico «A. I. Hertzen».

OGLIVE, J.F. (1990). The Nature of the Chemical Bond. Journal of the Chemical Education, 67, pp. 280-289.

PAULING, L, (1992). The Nature of the Chemical Bond. Journal of the Chemical Education, 69(7), pp. 519-521.

PÉREZ MATOS, R. y NOVOA CASTIEL, A. (1993). Predicción de estructuras moleculares. Santiago de Cuba: Impresión Ligera del Instituto Superior Pedagógico «Frank País».

RIERA, A. (ed.) (1989). Modelos teóricos e informáticos en la química actual. Madrid: Fundación Ramón Areces.

ROBERTS, J. D. (1969). Cálculos con orbitales moleculares. Barcelona: Reverté.

SANDERSON, R.T. (1960). Periodicidad química. Madrid: Aguilar.

SEGURA, D. (1991). Una premisa para el cambio conceptual: el cambio metodológico. Enseñanza de las Ciencias, 9 (2), pp. 175-180. 


\section{ANEXO I}

\section{ESQUEMA DE CONTENIDOS PARA EL TRATAMIENTO CLÁSICO DE LA ESTRUCTURA DE LA SUSTANCIA}

El aspecto estructural de la reacción química. Generalidades. Estructura - interacción (movimiento) - energía (media del movimiento). Estructura molecular y enlace. Estructura y geometría moleculares. Relación: estructura - propiedades aplicaciones. El cambio estructural en la reacción química, su descripción energética. Niveles estructurales de la sustancia: el atómico, el molecular y el intermolecular.

El nivel atómico de la sustancia. El atomismo hasta BohrSommerfeld. Modelos atómicos. El átomo como portador material de las propiedades de los elementos químicos. Radio atómico. La ionización de átomo, su aspecto energético: energía de ionización y afinidad electrónica. Radio iónico. La electronegatividad del átomo aislado: escala de Patsing.

La ley periódica de los elementos químicos. Tabla periódica. Variación periódica de las propiedades de los químicos.

El nivel molecular de la sustancia. El enlace químico: definición. Moléculas, iones y radicales. Historia de las nociones sobre el enlace químico y la valencia. Enlace químico e interacción atómica, mecanismo de formación del enlace. Generalidades, descripción energética. Predicción de la topología molecular*. Características del enlace químico: direccionalidad, fuerza y flexibilidad. Parámetros que caracterizan la geometría molecular: distancia y ángulo de enlace, ángulo diedro. Energía de enlace.

Electronegatividad de los átomos en el enlace. Modelo de Sanderson. Factores diferenciantes del enlace químico: la naturaleza de los átomos que se enlazan y la localización de los electrones del eniace. La polaridad del enlace y la conjugación de los electrones del enlace. Polaridad del enlace é indices para su evaluación. Cargas efectivas. Modelos límites del enlace químico covalente, iónico y metálico. Sentido físico de los modelos.

Sustancias moleculares. Propiedades generales. Naturaleza de las fuerzas interatómicas. El enlace covalente, su interpretación

* Aquí el término topología se adopta para referir la forma en que se concatenan entre sí los átomos en una molécula (Pérez Matos y Novoa Castiel, 1993. clásica. Enfoque electrónico del enlace covalente. Modelo del Lewis. Geometría de la sustancias moleculares (tipos). Teoría de la repulsión de los pares electrónicos. La isomería en los compuestos químicos. Las particularidades estructurales de los compuestos del carbono.

Sustancias iónicas. Propiedades generales. Naturaleza química del enlace iónico. Aspecto energético del enlace iónico. Energía reticular.

Sustancias atómicas. Cristales atómicos metálicos y no metálicos. El modelo de la atmósfera electrónica para la descripción del enlace metálico. El modelo del sólido extendido.

El estado sólido. Cuerpos sólidos cristalinos y amorfos. Definición de red cristalina y espacial. Elementos de una red espacial. Diferentes tipos de redes de acuerdo con la naturaleza de las redes estructurales. Parámetros estructurales de la celda. Sistemas cristalinos. Isotropía y anisotropia. Clasificación de las redes según la posición de las unidades estructurales. Propiedades. Isomorfismo y polimorfismo. Concepto de cristal. Elementos geométricos del cristal y su relación con la estructura interna. Defectos presentes en las estructuras cristalinas. Cristalización.

Fuerzas intermoleculares y agregación molecular. Naturaleza de las fuerzas intermoleculares. Significación de las fuerzas intermoleculares en la determinación de las propiedades físicas y químico-físicas de las sustancias. Diferentes tipos de fuerzas intermoleculares: de orientación, dispersivas, formación de dipolos e interacción dipolo-dipolo. Vector momento dipolo. Influencia de la polaridad en la magnitud de las fuerzas intermoleculares. Polarizabilidad de las moléculas e iones. Enlaces por puentes. Enlace por puente de bidrógeno.

El estado líquido đe agregación. Estructura de los líquidos. Interacción soluto-disolvente para solutos cristalinos (ínicos y covalentes) y moleculares. 
ANEXO II

\section{ESQUEMA DE CONTENIDOS PARA EL TRATAMIENTO CUÁNTICO DE LA ESTRUCTURA DE LA SUSTANCIA}

Evidencias experimentales del comportamiento cuántico de los microsistemas. Contenido y forma de la mecánica cuántica. Diferencia formal con la mecánica clásica.

La interacción como forma de movimiento en el micromundo. Sistema cuántico. La partícula en el pozo de potenciales como modelo del electrón en átomos y moléculas. La descripción del movimento en sistemas cuánticos, particularidades: cuantización (la cuantización de la energia y la geometría) e indeterminación. La descripción del estado cuántico: $\Psi$. El principio de superposición de los estados. Las ecuaciones de Schrödinger dependiente e independiente del tiempo. Los estados estacionarios. El formalísmo matemático de la mecánica cuántica: los operadores y la función de onda. Contenido físico. Sentido físico de $\Psi . \Psi *$. La conmutación de operadores y los sistemas completos de magnitudes en la descripción de los sistemas cuánticos. Los postulados de la mecánica cuántica.

Soluciones de la ecuación de Schgrödinger independiente del tiempo para sistemas hidrogenoides. Descripción cualitativa. $\Psi$ y los orbitales atómicos. Factirización de $\Psi$ : función radial $y$ angular.

Átomosmultielectrónicos. Características físicas de la interacción en átomos multielectrónicos: la ruptura de la degeneración de $n$. El espín electrónico. La solución de la ecuación de Schrödinger en átomos multielectrónicos. Aproximación de BornOppenheimer. Métodos aproximados para la resolución de la ecuación de Schrödinger. El método de Hartree-Fock. Orbitales de Slater: $n^{*}, z^{*} \delta$. Reglas de Slater.
Periodicidad de los elementos y distribución electrónica. Ley periódica. Esquema energético de los orbitales atómicos. Estados electrónicos excitados. Espectros atómicos.

Descripción cuántica del dominio molecular. La combinación lineal de orbitales atómicos (CLOA) como artificio mecanocuántico para la descripción del enlace, sentido físico. Condiciones para la combinación efectiva de orbitaies atómicos. El orbital molecular. Las fuerzas de intercambio y la unión química.

Teoría del enlace de valencia. Principios. Los orbitales híbridos. Hibridación. La resonancia como solución al problema de la delocalizacíón electrónica en los marcos de la TEV.

Teoria de los orbitales moleculares. Principios y formulaciones generales. Los orbitales moleculares dirigidos como solución al problema de la direccionalidad del enlace covalente en la TOM. Estabilidad (fortaleza) y orden de enlace. Moléculas diatómicas homonucleares y heteronucleares. Las moléculas poliatómicas. Los sistemas mecano-cuánticos decălculo molecular. Diagramas energéticos de O.M. Orbitales fronteras. La optimitización de geometría. El cálculo de los estados electrónicos excitados. Espectros electrónicos.

La cuantización de los movimientos vibracionales y rotacionales. Los espectros vibracionales y rotacionales. Los espectros vibracionales. Espectroescopía infrarroja. Generalidades.

La teoría de las bandas en sólidos extendidos. Nivel de Fermi. La cuantización de las fuerzas de Van der Waals. Generalidades. 
